EPJ Web of Conferences 81, 04010 (2014)

DOI: $10.1051 /$ epjconf/ 20148104010

(C) Owned by the authors, published by EDP Sciences, 2014

\title{
Recent results on B and D decays from $\mathrm{LHCb}$
}

\author{
Agnieszka Oblakowska-Mucha ${ }^{1, a}$ \\ for the LHCb Collaboration \\ ${ }^{1}$ AGH University of Science and Technology, Faculty of Physics and Applied Computer Science \\ Al.Mickiewicza 30, 30-059 Krakow, Poland
}

\begin{abstract}
LHCb spectrometer collected more than $3 \mathrm{fb}^{-1}$ of data in the years 2010-12. This brief summary presents the very latest results obtained in the rare B decays, mixing and $C P$ violation in charm sector.
\end{abstract}

\section{LHCb spectrometer}

$\mathrm{LHCb}$ (figure 1) is a dedicated experiment for the study of flavour physics at the LHC [1]. In particular the experiment is designed to study $C P$ violation, rare beauty and charm particles decays and searches for New Physics evidences. The heavy quarks pairs in proton-proton collisions are produced mainly in the forward region so the $\mathrm{LHCb}$ is a single-arm forward spectrometer covering the pseudorapidity range $2<\eta<5$. The spectrometer includes a high-precision tracking system which enables a momentum resolution $\Delta p / p$ that varies from $0.4 \%$ at $5 \mathrm{GeV} / c$ to $0.6 \%$ at $100 \mathrm{GeV} / c$, and an impact parameter (IP) resolution of $12 \mu \mathrm{m}$ for tracks with high transverse momentum. Particle identification in wide momentum range with $95 \%$ efficiency is provided by two RICH detectors. The trigger consists of a hardware stage, based on information from the calorimeter and muon systems, followed by a software stage which applies a full event reconstruction. The trigger reduces $11 \mathrm{MHz}$ of bunch-bunch crossings with inelastic collisions to a rate of $3-5 \mathrm{kHz}$, which is written

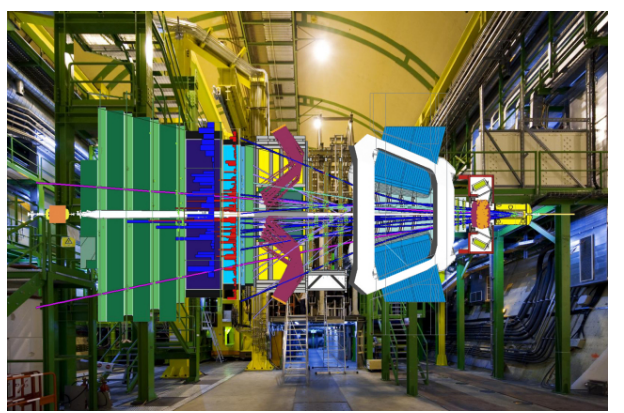

Figure 1. The LHCb spectrometer. to storage. More then $3 \mathrm{fb}^{-1}$ have been recorded during the years 2010-12.

The LHCb physics program covers a wide range of $\mathrm{c}$ - and b-meson topics focused on both direct and indirect $\mathrm{CP}$ violation, spectroscopy, rare decays and many others.

\section{Rare $B$ meson decays as a probe for New Physics}

Rare decays of $b$ and $c$ hadrons are suppressed or forbidden in the SM and thus constitute a very sensitive environment for the study of New Physics (NP). Since they mostly occur via loop diagrams,

\footnotetext{
ae-mail: Agnieszka.Oblakowska-Mucha@cern.ch
} 
new massive particles could affect these loops and modify sizes of branching fractions, angular distributions of decay products or $C P$ asymmetries.

\subsection{Decay $B \rightarrow K^{*} \mu^{+} \mu^{-}$}

The $B \rightarrow K^{*} \mu^{+} \mu^{-}$decay is very rich in observables, which can help in constraining the size of NP. $\mathrm{LHCb}$ presents the analysis of: differential branching ratios, angular branching fractions and forwardbackward asymmetry, in six bins of $q^{2}$. The first measurement of zero-crossing point has been performed, yielding $q_{0}^{2}=\left(4.9_{-1.3}^{+1.1}\right) \mathrm{GeV}^{2} / c^{4}$ [2]. This result and $C P$ asymmetry are consistent with the SM prediction.

Angular distributions are functions of the Wilson coefficients, which contain information about short distance effects (sensitive to physics beyond the SM) and form factors (depended on long distance effects). In general, the measurements agree with SM expectations (figure 2a), apart from a sizeable discrepancy in the interval $4.30<q^{2}<8.68 \mathrm{GeV}^{2} / c^{4}$ for the observable $P_{5}^{\prime}$, see figure $2 \mathrm{~b}$. Measurements with more data and further theoretical studies [3] will be important to draw more definitive conclusions about this fenomena.
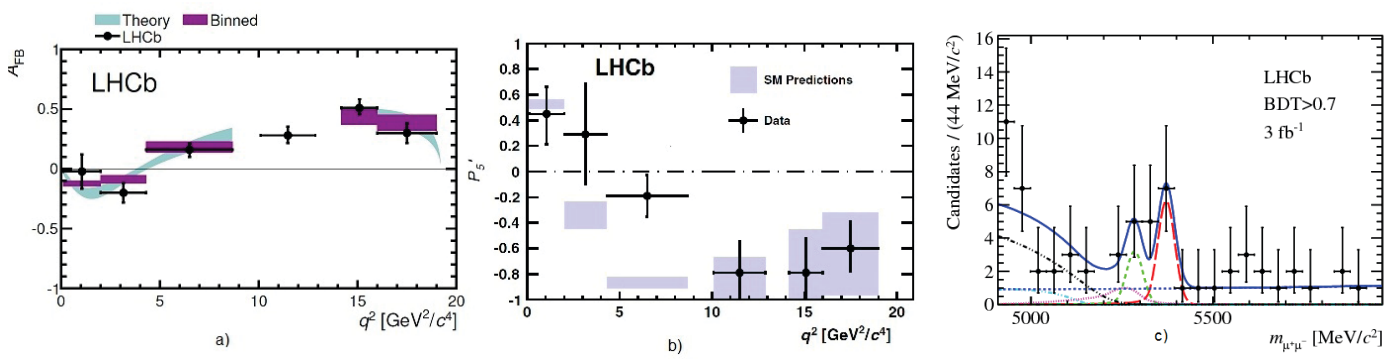

Figure 2. a) Distribution of forward-backward asymmetry [2], b) $P_{5}^{\prime}$ coefficient [4], c) invariant mass distribution of $B_{s} \rightarrow \mu^{+} \mu^{-}$selected events [5].

\subsection{Decay $B_{(S)} \rightarrow \mu^{+} \mu^{-}$}

The $B \rightarrow \mu^{+} \mu^{-}$decays are flavour changing neutral currents (FCNC) and therefore are only allowed at loop level within the SM. In addition, they are further suppressed by the the GIM mechanism and by their helicity structure. The analysis is performed on the $3.1 \mathrm{fb}^{-1}$ data sample collected in 2011-12 at 7-8 $\mathrm{TeV}$ center of mass energy. The main discrimination between the signal and combinatorial background is brought by the BDT. A simultaneous unbinned maximum likelihood fit to the mass projections in the BDT bins is performed to extract the signal distribution (figure $2 \mathrm{c}$ ). This is the first evidence for the $B_{s} \rightarrow \mu^{+} \mu^{-}$decay, which observed branching fraction is: $B R\left(B_{s} \rightarrow \mu^{+} \mu^{-}\right)=$ $\left(2.9_{-1.2}^{+1.5}\right) \times 10^{9}$, consistent with the SM expectation. In the case of $B \rightarrow \mu^{+} \mu^{-}$, the data in the signal mass window are consistent with the background expectation and the world's best upper limit is set at $B R\left(B \rightarrow \mu^{+} \mu^{-}\right)<7.4 \times 10^{10}$ at $95 \%$ C.L. [5].

\section{Latest results in charm sector}

\subsection{Mixing and indirect CP violation in $D^{0}$ decays}

The mass eigenstates of the neutral charm meson system $\left|D_{1,2}\right\rangle$, with masses $m_{1,2}$ and decay widths $\Gamma_{1,2}$, can be represented as linear combination of the flavour eigenstates $\left|D^{0}\right\rangle$ and $\left|\bar{D}^{0}\right\rangle$ as a $\left|D_{1,2}\right\rangle=$ 

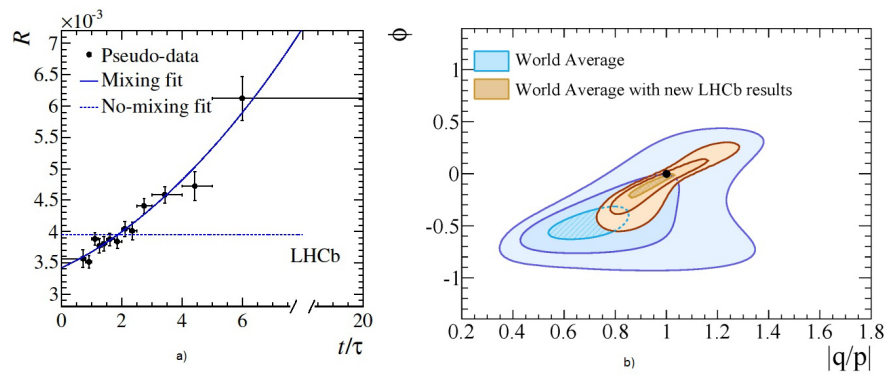

Figure 3. a) Decay-time evolution of the ratio $R$, of $W S$ to $R S$ yields (see [6] for detailed explanation), b) $|q / p|$ value from [7].

$p\left|D^{0}\right\rangle \pm q\left|\bar{D}^{0}\right\rangle$ with complex coefficients $|p|^{2}+|q|^{2}=1$. This allows to define the mixing parameters: $x \equiv 2\left(m_{1}+m_{2}\right) /\left(\Gamma_{1}+\Gamma_{2}\right)$ and $\left.y \equiv\left(\Gamma_{2}-\Gamma_{1}\right) / \Gamma_{1}+\Gamma_{2}\right)$. The LHCb collaboration has observed mixing for the first time using $D^{0}$ decays $D^{0} \rightarrow K^{-} \pi^{+}$(which proceeds mainly through Cabbibo-favoured CF tree-level Right-Sign decay) or decay $D^{0} \rightarrow K^{+} \pi^{-}$, which can occur either through a doubly Cabbibosuppressed (DCS) decay or through a mixing of the $D^{0}$ into $\bar{D}^{0}$ followed by a CF decay (Wrong-Sign decay). The time-dependent ratio $R$ of $W S$ to $R S$ decay rates that depends on the mixing parameters $x, y$ is shown on figure $3 \mathrm{a}$ and the most precise determiantion of $|p / q|$ on figure $3 b$.

The results from the whole Run I dataset of $3.1 \mathrm{fb}^{-1}$ show no indication of $C P$ asymmetry in mixing nor in the interference between mixing and decay amplitudes [7].

\subsection{Direct CP violation}

The evidence for the direct $\mathrm{CP}$ violation has been measured as a difference of asymmetries in the timeintegrated decays rates of $D^{0}$ to $K^{+} K^{-}$or $\pi^{+} \pi^{-}$final states defining $\Delta A_{C P}=A_{C P}\left(K^{+} K^{-}\right)-A_{C P}\left(\pi^{+} \pi^{-}\right)$. While the initial evidence for $C P$ violation has bee shown by LHCb [8], the most recent updates has not confirmed this effect [9]. The current world average is $\Delta A_{C P}=(-0.333 \pm 1.20) \%$.

\section{Conclusions}

LHCb has proven its potential to obtain valuable result in both $c$ and $b$ hadron sector. The results, in most of the cases, are compatible with the predictions of the Standard Model. LHCb has observed very rare decays never observed before or pushed further the limits on new observables with greatly improved precision.

\section{References}

[1] The LHCb Collaboration, JINST 3 (2008), S08005

[2] The LHCb Collaboration, JHEP 1308 (2013), 131

[3] S.Descotes-Genon, T.Hurth, J.Matias, JHEP 1305 (2013), 137, Phys.Rev.D 88, 074002 (2013)

[4] R.Aaij et al., Phys.Rev.Lett. 111 (2013), 191801

[5] R.Aaij et al., Phys.Rev.Lett 111 (2013), 101805

[6] R.Aaij et al., Phys. Rev. Lett. 110 (2013), 101802

[7] R.Aaij et al., Phys. Rev. Lett. 111 (2013), 251801

[8] R.Aaij et al., Phys.Rev.Lett 108 (2012), 111602

[9] R.Aaij et al., CERN-LHCb-CONF-2013-003. R.Aaij et al., Phys.Rev.Lett. 110 (2013), 101802 